\title{
Mirtrons as a possible inherent source of silencing variability
}

\author{
P.S. Vorozheykin ${ }^{1}$, I.I. Titov ${ }^{1,2} *$ \\ ${ }^{1}$ Novosibirsk State University, Novosibirsk, Russia \\ ${ }^{2}$ Institute of Cytology and Genetics SB RAS, Novosibirsk, Russia \\ *e-mail:titov@bionet.nsc.ru
}

Key words: miRNA, introns, secondary structure, mirtrons

Background: MicroRNAs proceeds through the different canonical and non-canonical pathways; the most frequent of the non-canonical ones is the splicing-dependent biogenesis of mirtrons. We compare the mirtrons and non-mirtrons of human and mouse to explore how their maturation appears in the precursor structure around the miRNA [1]. Results: A typical structure of the annotated mirtron pre-miRNAs differs from the canonical pre-miRNA structure and possesses the 1- and $2 \mathrm{nt}$ hanging ends at the hairpin base. The mirtron Dicer cleavage site shows the excessive variability (partially due to guanine at its ends inherited from splicing) than the canonical one. In contrast with the canonical miRNAs the mirtrons have higher SNP densities and their pre-miRNAs are inversely associated with diseases. Therefore we supported the view that mirtrons are under positive selection while canonical miRNAs are under negative one and we suggested that mirtrons are an intrinsic source of silencing variability which produces the disease-promoting variants. Finally, we considered the interference of the pre-miRNA structure and the U2snRNA:pre-mRNA basepairing. We analyzed the location of the branchpoints and found that mirtron structure tends to expose the branchpoint site what suggests that the mirtrons can readily evolve from occasional hairpins in the immediate neighbourhood of the $3^{\prime}$ splice site.

Conclusion: The miRNA biogenesis manifests itself in the footprints of the secondary structure. Close inspection of these structural properties can help to uncover new pathways of miRNA biogenesis and to refine the known miRNA data, in particular, new non-canonical miRNAs may be predicted or the known miRNAs can be re-classified.

\section{References}

1. Titov I.I., Vorozheykin P.S. (2018) Comparing miRNA structure of mirtrons and non-mirtrons. BMC Genomics. 19(Suppl.3):114. 